\title{
LncRNA UCA1 promotes cell proliferation, invasion and migration of laryngeal squamous cell carcinoma cells by activating $\mathbf{W n t} / \boldsymbol{\beta}$-catenin signaling pathway
}

\author{
SUGUANG SUN, CHENG GONG and KUN YUAN \\ Department of Otolaryngology-Head and Neck Surgery, The Central Hospital of Wuhan, Tongji Medical College, \\ Huazhong University of Science and Technology, Wuhan, Hubei 430014, P.R. China
}

Received February 1, 2018; Accepted July 31, 2018

DOI: $10.3892 /$ etm.2018.7097

\begin{abstract}
In view of the poor prognosis of laryngeal squamous cell carcinoma (LSCC) and the functionality of long non-coding (lnc)RNA UCA1 in different types of cancer, the present study aimed to investigate the role of UCA1 in the development and progression of LSCC. A total of 90 patients with LSCC and 90 healthy subjects were enrolled in the present study. Expression levels of UCA1 in tumor tissues and adjacent healthy tissues, as well as serum of patients with LSCC and normal controls were detected by reverse transcription-quantitative polymerase chain reaction. Receiver operating characteristic curve analysis was performed to evaluate the diagnostic value of serum UCA1 for LSCC. Survival curves were plotted using the Kaplan-Meier method and employed to evaluate the prognosic values of serum UCA1 for LSCC. Cell proliferation, migration and invasion were detected using the cell proliferation assay, and Transwell migration and invasion assays, respectively. Expression levels of $\mathrm{Wnt} / \beta$-catenin-associated proteins were detected by western blot analysis. Results indicated that the expression levels of UCA1 were significantly higher in tumor tissues compared with adjacent healthy tissues in the majority of patients with LSCC. In addition, serum levels of UCA1 were significantly higher in patients with LSCC coapred with healthy controls. UCA1 overexpression promoted, whereas UCA1 knockdown inhibited the proliferation, migration and invasion of LSCC cells. UCA1 overexpression activated the Wnt/ $\beta$-catenin signaling pathway in LSCC cells, whereas treatment with Wnt inhibitor reduced the enhancing effects of UCA1 overexpression on the proliferation, migration and
\end{abstract}

Correspondence to: Dr Kun Yuan, Department of Otolaryngology-Head and Neck Surgery, The Central Hospital of Wuhan, Tongji Medical College, Huazhong University of Science and Technology, 26 Victory Street, Wuhan, Hubei 430014, P.R. China

E-mail: hlui3k0o@yeah.net

Key words: UCA1, laryngeal squamous cell carcinoma, proliferation, migration, invasion, $\mathrm{Wnt} / \beta$-catenin signaling pathway invasion of LSCC cells. The present findings suggest that UCA1 can promote cell proliferation, invasion and migration of LSCC cells by activating the Wnt/ $\beta$-catenin signaling pathway.

\section{Introduction}

As one of the most aggressive neoplasms of the neck and head, laryngeal squamous cell carcinoma (LSCC) is a deadly disease that affects $>20,000$ people in the United States every year (1). Although the incidence of LSCC is low compared with other types of cancer, LSCC is now considered to be one of the major causes of cancer-associated fatalities worldwide, particularly in China (2). Despite the progress in the treatment of LSCC, the treatment outcomes of patients remain poor, particularly for patients in advanced stages (3). Therefore, early diagnosis and treatment is critical to improve the survival rates of patients with LSCC.

Long non-coding (lnc)RNAs, refer to a group of RNA transcripts containing $>200$ nucleotides that are not translated into protein products (4). LncRNAs achieve their biological functions through multiple pathways to participate in almost all of the critical biochemical and physiological processes in the human body (5). Furthermore, IncRNAs have important functions in the development of human diseases, including different types of cancer (6). It has been well established that lncRNA UCA1 can serve an oncogenic role in the development and progression of several types of malignancies (7). However, the functionality of UCA1 in LSCC remains unclear. Activation of the Wnt/ $\beta$-catenin signaling pathway contributes to the growth and metastasis of laryngeal cancer (8). Notably, previous findings have suggested that UCA1 can achieve its biological roles through interactions with the $\mathrm{Wnt} / \beta$-catenin signaling pathway (9). Therefore, it is be reasonable to hypothesize that UCA1 may also interact with $\mathrm{Wnt} / \beta$-catenin to participate in LSCC.

In the present study, expression levels of UCA1 in tumor tissues and adjacent healthy tissues of patients with LSCC, as well as in the serum of patients with LSCC and healthy controls were measured. Effects of UCA1 knockdown and overexpression on the proliferation, migration and invasion of LSCC cells were explored. The potential of serum UCA1 to 
serve as a diagnostic and prognostic marker for LSCC was investigated. In addition, interactions between UCA1 and Wnt/ $\beta$-catenin signaling pathway, a key pathway that involved in tumorigenesis (8), were also studied.

\section{Materials and methods}

Patients. Between July 2008 and July 2010, a total of 90 patients (41 males and 49 females; age range, 22-71 years; mean age, $49 \pm 14.3$ years) with LSCC in the Central Hospital of Wuhan (Wuhan, China) were selected to serve as research subjects. Patients were included if they were diagnosed with LSCC via pathological examinations and if they were willing to participate. Patients were excluded if they had any other disease or if they received treatment within 3 months prior to admission. All patients were diagnosed with LSCC by imaging tests and pathological examinations. Distant tumor metastasis were identified in 51 patients. All patients received surgical resection, and tumor tissues and adjacent healthy tissues within $0.5 \mathrm{~cm}$ surrounding the tumors were collected during surgical resection. At the same time, a total of 90 healthy subjects (48 males and 42 females; age range, 26-75 years old, with a mean age of $51 \pm 17.9$ years) were also selected to serve as the healthy control group. There were no significant differences in age and sex between the patient group and control group. The Ethics Committee in the Central Hospital of Wuhan approved the present study, and all patients had signed an informed consent statement.

Preparation of serum samples. Fasting blood $(10 \mathrm{ml})$ was extracted from each participant in the morning. Blood was transferred to non-anticoagulation tubes and maintained at room temperature for $1 \mathrm{~h}$ for hemolysis, followed by centrifugation at $1,000 \mathrm{x}$ g for $15 \mathrm{~min}$ at room temperature to collected serum.

Cell line, cell culture and transfection. The human LSCC cell line AMC-HN-8 was purchased from Otwo Biotech Inc. (Shenzhen, China). Cells were cultured in Dulbecco's Modified Eagle Medium supplemented with $10 \%$ fetal bovine serum. Cells were harvested at the logarithmic growth phase. UCA1 small interfering (si)RNA (cat. no. 4390771) and Silencer Select Negative Control No. 2 siRNA were purchased from Thermo Fisher Scientific, Inc. (Waltham, MA, USA) to establish the AMC-HN-8 UCA1 siRNA silencing cell line. The full length UCA1 cDNA was inserted into a pIRSE2-EGFP vector (Clontech Laboratories, Inc., Mountainview, CA, USA) to establish UCA1 expression vector. Cells were cultured overnight until 80-90\% confluency was reached. Lipofectamine 2000 reagent (cat. no. 11668-019; Invitrogen; Thermo Fisher Scientific, Inc.) was used to transfect UCA1 siRNA and UCA1 expression vector into AMC-HN-8 cells. Transfection with Silencer Select Negative Control No. 2 siRNA and empty pIRSE2-EGFP vector was used as negative control.

Cell proliferation assay. Cells were harvested at the logarithmic growth phase and used to prepare single cell suspensions. Cell density was adjusted and cells were transferred into 96-well plates at a density of $4 \times 10^{3}$ cells per well. Cells were cultured under normal conditions $\left(37^{\circ} \mathrm{C}, 5 \% \mathrm{CO}_{2}\right)$, and $10 \mu \mathrm{l}$ of Cell
Counting Kit-8 (CCK-8) solution (Sigma-Aldrich, Merck KGaA, Darmstadt, Germany) was added at 24, 4872 and $96 \mathrm{~h}$ later. Incubation was performed for a further $5 \mathrm{~h}$ and the optical density values $(450 \mathrm{~nm})$ were measured using a microplate reader. UCA1 knockdown and overexpression was detected prior to and post-experiments to ensure knockdown and overexpression rates were $>50 \%$ during the whole procedure. In cases of Wnt inhibitor treatment, cells were pretreated with Wnt inhibitor IWP-2 (Sigma-Aldrich; Merck KGaA) at a dose of $2 \mu \mathrm{g} / \mathrm{ml} 24 \mathrm{~h}$ prior to use.

Cell migration and invasion assays. Transwell cell migration and invasion assays were performed to evaluate cell migration and invasion abilities. All reagents used were provided by BD Biosciences (San Jose, CA, USA). In the migration assay, $4 \times 10^{4}$ cells were transferred to the upper chamber, while RPMI-1640 medium (Thermo Fisher Scientific, Inc.) supplemented with $20 \%$ fetal calf serum (Sigma-Aldrich; Merck $\mathrm{KGaA}$ ) was used to fill the lower chamber. Cells were incubated under normal conditions before the membranes were cleaned and stained with $0.5 \%$ crystal violet (Sigma-Aldrich; Merck KGaA) for $20 \mathrm{~min}$ at room temperature. Stained cells were counted under an optical microscope (Olympus Corporation, Tokyo, Japan). Prior to the invasion assay, the upper chamber was pre-coated with Matrigel (cat. no. 356234, EMD Millipore, Billerica, MA, USA). UCA1 knockdown and overexpression were detected prior to and post-experiments to ensure knockdown and overexpression rates were $>50 \%$ during the whole procedure. In cases of Wnt inhibitor treatment, cells were pretreated with Wnt inhibitor IWP-2 at a dose of $2 \mu \mathrm{g} / \mathrm{ml} 24 \mathrm{~h}$ prior to use.

Reverse transcription-quantitative polymerase chain reaction (RT-qPCR). Total RNA extraction from tumor tissues, adjacent healthy tissues, serum and in vitro cultured cells was performed using TRIizol reagent (Invitrogen; Thermo Fisher Scientific, Inc.). RNA quality was examined using a NanoDrop 2000 spectrophotometer (Thermo Fisher Scientific, Inc.). RNA samples with a A260/A280 ratio between 1.8 and 2.0 were used in RT to synthesize cDNA. RT was performed using High-Capacity cDNA Reverse Transcription kit (Thermo Fisher Scientific., Inc.) according to manufacturer's protocol. Reaction conditions were as follows: $5 \mathrm{~min}$ at $25^{\circ} \mathrm{C}, 20 \mathrm{~min}$ at $50^{\circ} \mathrm{C}$ and $5 \mathrm{~min}$ at $75^{\circ} \mathrm{C}$. A SYBR ${ }^{\circledR}$ Green Quantitative RT-qPCR kit (Sigma-Aldrich; Merck KGaA) was utilized to prepare all PCR reaction systems. The following primers were used in PCR reactions: UCA1, forward 5'-CCCAAG GAACATCTCACCAATT-3' and reverse 5'-TGAGGGGTC AGACTTTTGACAAG-3'; and $\beta$-actin, forward 5'-GACCTC TATGCCAACACAGT-3' and reverse 5'-AGTACTTGCGCT CAGGAGGA-3'. PCR reactions were performed on an ABI PRISM 7500 sequence detection system (Applied Biosystems; Thermo Fisher Scientific, Inc.). PCR reaction conditions were as follow: $95^{\circ} \mathrm{C}$ for $36 \mathrm{sec}$, followed by 40 cycles of $95^{\circ} \mathrm{C}$ for $12 \mathrm{sec}$ and $60^{\circ} \mathrm{C}$ for $42 \mathrm{sec}$. Expression levels were quantified using the $2^{-\Delta \Delta \mathrm{Cq}}$ method (10), and expression levels of UCA1 were normalized to endogenous control $\beta$-actin.

Western blot analysis. Following total protein extraction from in vitro cultured cells using cell lysis buffer (Clontech 
Laboratories, Inc.). The bicinchoninic acid assay was performed to determine protein quality. Subsequently, $10 \%$ SDS-PAGE was performed using $20 \mu \mathrm{g}$ of protein from each protein sample. The separated proteins were transferred onto polyvinylidene difluoride membranes (Bio-Rad Laboratories, Inc., Hercules, CA, USA). Membranes were blocked with $5 \%$ non-fat milk for $2 \mathrm{~h}$ at room temperature. The membranes were washed 3 times with PBS for $10 \mathrm{~min}$ each and incubated with corresponding primary antibodies, including rabbit anti-p-glycogen synthase kinase (GSK)-3 $\beta$ antibody (1:2,000; cat. no. ab32391; Abcam, Cambridge, UK), anti-GSK-3 $\beta$ (Ser9) antibody (1:2,000; cat. no. ab75745; Abcam), anti- $\beta$-catenin antibody $(1: 2,000$; cat. no. ab32572; Abcam) and anti-GAPDH antibody (1:1,000; cat. no. ab9485; Abcam) overnight at $4^{\circ} \mathrm{C}$. The following day, membranes were washed and incubated with anti-rabbit IgG-horseradish peroxidase secondary antibody (1:1,000; cat. no. MBS435036; MyBioSource, Inc., San Diego, CA, USA) at room temperature for $1 \mathrm{~h}$. Membranes were washed again and signal development was performed using the enhanced chemiluminescence kit (Sigma-Aldrich; Merck KGaA) method. Relative expression levels of each protein were normalized to endogenous control GAPDH using ImageJ v1.46 software (National Institutes of Health, Bethesda, MD, USA).

Statistical analysis. All statistical analyses were performed using SPSS19.0 (IBM Corp., Armonk, NY, USA). Normal distribution data were expressed as the mean \pm standard deviation of the mean. Differences between groups were compared using a Student's t-test or one-way analysis of variance followed by a Least Significant Difference post hoc test, accordingly. Non-normal distribution data were compared using the non-parametric Mann-Whitney U test. According to the median serum level of UCA1, 90 patients with LSCC were divided into a high expression group $(\mathrm{n}=45)$ and a low expression group $(\mathrm{n}=45)$. Survival curves were plotted for each group using the Kaplan-Meier method and compared using a $\log$ rank t-test to assess the prognosic values of serum UCA1 for LSCC. $\mathrm{P}<0.05$ was considered to represent a statistically significant difference.

\section{Results}

Expression of UCA1 in tumor tissues and adjacent healthy tissues of LSCC patients. LncRNA UCA1 commonly acts as an oncogene and its expression is increased in different types of tumors (7). Therefore, the expression levels of UCA1 in tumor tissues and adjacent healthy tissues of 90 patients with LSCC were detected using RT-qPCR. As indicated in Fig. 1, the expression levels of UCA1 were significantly higher in tumor tissues compared with adjacent healthy tissues in $80 / 90$ patients with LSCC $(\mathrm{P}<0.05)$. These data suggest that upregulated expression of UCA1 may likely be involved in the development of LSCC.

Serum levels of UCA1 in patients with LSCC and healthy controls with the diagnostic values for LSCC. Serum levels of UCA1 in 90 patients with LSCC and 90 healthy controls were measured using RT-qPCR. As indicated in Fig. 2A, serum levels of UCA1 were significantly higher in patients with LSCC compared with healthy controls. ROC curve analysis (11) was used to evaluate the diagnostic values of UCA1 serum levels for LSCC. As indicated in Fig. 2B, the area under the curve was 0.8905 , with a $95 \%$ confidence interval of 0.8408 to $0.9402(\mathrm{P}<0.0001)$. These results suggest that serum UCA1 may potentially serve as a diagnostic marker for LSCC.

Prognostic value of serum UCAl for LSCC. According to the median serum level of UCA1, 90 patients with LSCC were divided into a high expression group $(n=45)$ and a low expression group $(n=45)$. As indicated in Table I, serum levels of UCA1 exhibited no significant difference regarding sex, age and patients' living habits, such as smoking and drinking. However, high serum levels of UCA1 were indicated to be significantly associated with distant metastasis. Survival curves of patients with LSCC with high and low serum levels of UCA1 were plotted using the Kaplan-Meier method (Fig. 3). Results indicated that the overall survival rate of patients with high serum levels of UCA1 was significantly lower than that of patients with low serum levels of UCA1 ( $\mathrm{P}=0.032)$. The results suggest that increased serum levels of UCA1 in patients with LSCC is associated with poor prognosis.

Effects of UCA1 knockdown and overexpression on proliferation, migration and invasion of $\mathrm{LSCC}$ cell line $A M C-H N$-8. It is well known that UCA1 can promote the growth and metastasis of different types of cancer $(12,13)$. In the present study, UCA1 siRNA silencing and overexpression were first confirmed by RT-qPCR (Fig. 4A). Compared with control cells (C group), the proliferation ability of cells with UCA1 siRNA silencing was significantly reduced, whereas the proliferation ability of the AMC-HN-8 cells was significantly increased with UCA1 overexpression ( $\mathrm{P}<0.05$; Fig. 4B). Similarly, UCA1 siRNA silencing also significantly reduced the migration (Fig. 4C) and invasion (Fig. 4D) abilities of AMC-HN-8 cells $(\mathrm{P}<0.05)$, whereas UCA1 overexpression significantly promoted cell migration (Fig. 4C) and invasion ( $\mathrm{P}<0.05$; Fig. 4D). These data suggest that UCA1 may participate in LSCC by promoting tumor cell proliferation, migration and invasion.

Effects of UCA1 overexpression on the Wnt $/ \beta$-catenin signaling pathway in AMC-HN-8 cells. The Wnt/ $\beta$-catenin signaling pathway participates in the pathogenesis of various types of cancer (8). GSK-3 $\beta$ is a negative regulator of $\mathrm{Wnt} / \beta$-catenin signaling pathway (8). In the present study, no marked changes in the expression levels of GSK-3 $\beta$ were identified in AMC-HN-8 cells following UCA1 overexpression, whereas the phosphorylation level of GSK-3 $\beta$ (p-GSK-3 $\beta$ ) was significantly decreased in AMC-HN-8 cells with UCA1 overexpression compared with control cells (C group; $\mathrm{P}<0.05$; Fig. 5). By contrast, expression levels of $\beta$-catenin were significantly higher in AMC-HN-8 cells with UCA1 overexpression compared with control cells $(\mathrm{P}<0.05$; Fig. 5). These data suggest that UCA1 can activate the Wnt//-catenin signaling pathway to promote LSCC.

Effects of Wht inhibitor IWP-2 on proliferation, migration and invasion of AMC-HN-8 cell lines regarding UCAl expression. In the present study, UCA1 expression significantly promoted proliferation, migration and invasion of AMC-HN-8 cells, and activated the Wnt/ $\beta$-catenin signaling pathway. To further 


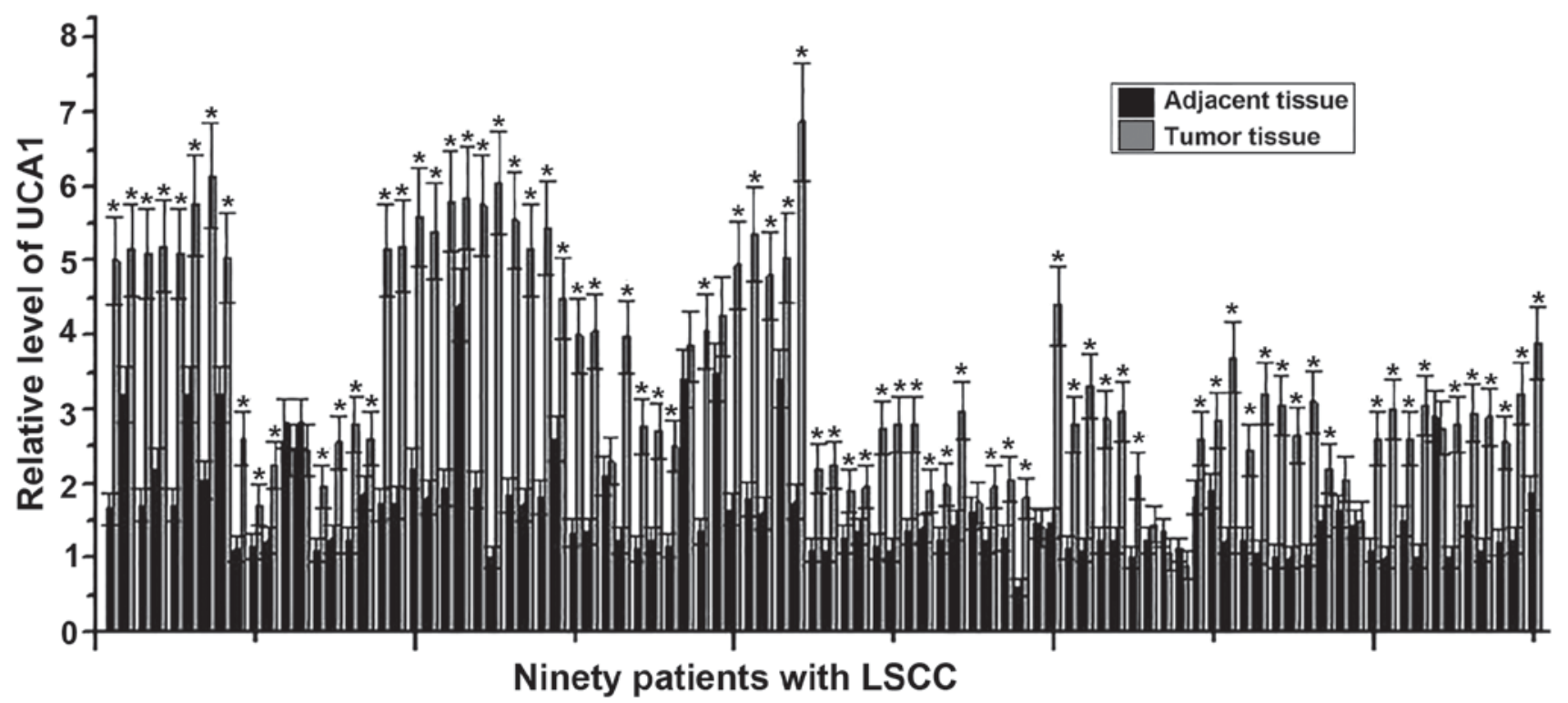

Figure 1. Expression of UCA1 in tumor tissues and adjacent healthy tissues of LSCC patients. The experiment was performed three times and data were expressed as mean \pm standard deviation of the mean. " $\mathrm{P}<0.05$, compared with adjacent healthy tissues. LSCC, laryngeal squamous cell carcinoma; UCA1, urothelial cancer associated 1 .
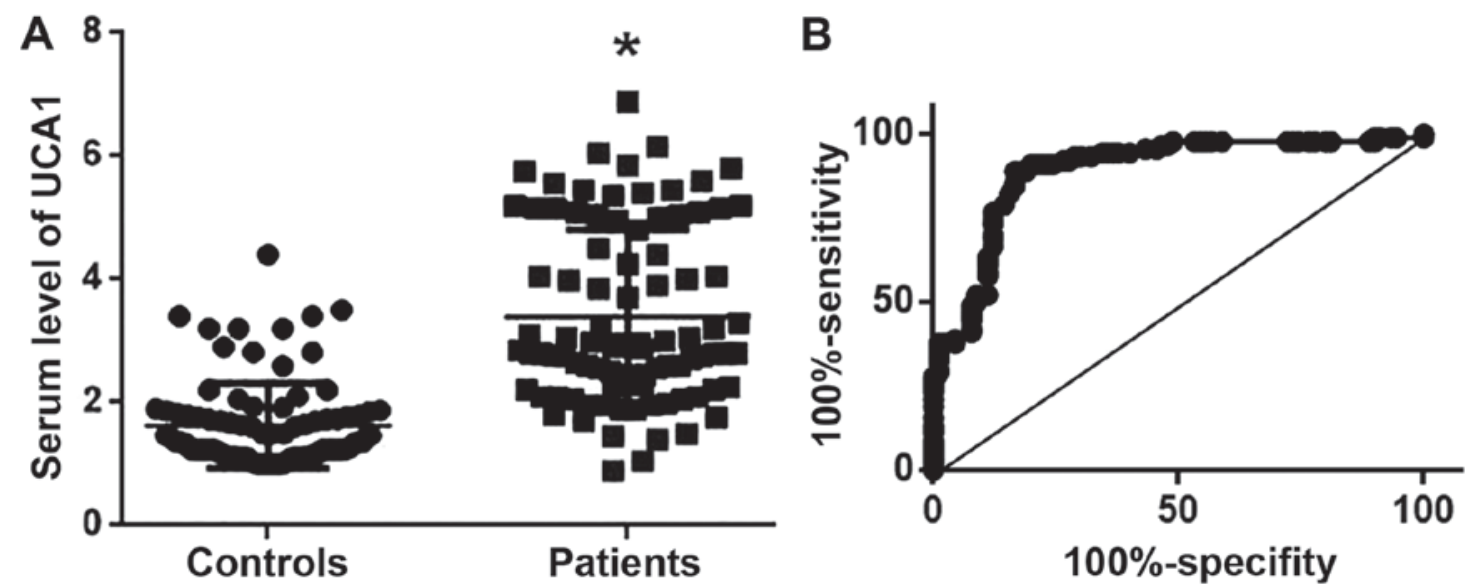

Figure 2. Serum levels of UCA1 and its diagnostic value for LSCC. (A) Comparison of serum levels of UCA1 in patients with LSCC and healthy controls; (B) Receiver operating characteristic curve analysis to evaluate the diagnostic value of serum UCA1 for LSCC. ${ }^{*}<0.05$, compared with healthy controls. LSCC, laryngeal squamous cell carcinoma; UCA1, urothelial cancer associated 1.

explore the interactions between UCA1 and Wnt/ $\beta$-catenin signaling, Wnt inhibitor IWP-2 (cat. no. sc-252928, Santa Cruz Biotechnology, Inc., Dallas, TX, USA) was used to treat UCA1-overexpressed AMC-HN-8 cells. As indicated in Fig. 6, IWP-2 treatment $\mathrm{U}(2 \mu \mathrm{g} / \mathrm{ml}$ for $24 \mathrm{~h})$ significantly reduced the enhancing effects of UCA1 overexpression on proliferation (Fig. 6A), migration (Fig. 6B) and invasion (Fig. 6C) of AMC-HN-8 cells $(\mathrm{P}<0.05)$. These results suggest that the enhancing effects of UCA1 overexpression on proliferation, migration and invasion of AMC-HN-8 cells may at least be partially dependent on the activation of Wnt/ $\beta$-catenin signaling pathway.

\section{Discussion}

LSCC is a rare but deadly malignancy. The development and progression of LSCC is a complex process involving various internal and external factors (14). Previous studies have revealed that smoking, drinking, laryngopharyngeal reflux and low body mass index are potential risk factors for LSCC (2). In spite of the progress in understanding the mechanisms of the LSCC, the etiology of this disease remains largely unknown (2). Recent studies have demonstrated that the development of LSCC is usually accompanied with changes in the genome-wide expression profile of lncRNAs, and some lncRNAs such as IncRNA HOTAIR have been proven to be key factors in the pathogenesis of this disease (15). LncRNA UCA1 is typically overexpressed in tumor tissues of different types of malignancies, which is consistent with its role in promoting the development and progression of those tumors (16). However, the expression pattern of lncRNA UCA1 in LSCC has not been reported. In the present study, expression levels of IncRNA UCA1 were identified to be significantly higher in tumor tissues compared with adjacent 
Table I. Comparison of clinical data of high and low UCA1 expression groups.

\begin{tabular}{|c|c|c|c|c|}
\hline Variables & Number of cases & High UCA1 expression & Low UCA1 expression & P-value \\
\hline \multicolumn{5}{|l|}{ Sex } \\
\hline Male & 41 & 20 & 21 & 0.29 \\
\hline Female & 49 & 25 & 24 & \\
\hline \multicolumn{5}{|l|}{ Age } \\
\hline$>50$ (years) & 42 & 19 & 23 & 0.17 \\
\hline$<50$ (years) & 48 & 26 & 22 & \\
\hline \multicolumn{5}{|l|}{ Smoker } \\
\hline Yes & 37 & 19 & 18 & 0.49 \\
\hline No & 53 & 26 & 27 & \\
\hline \multicolumn{5}{|l|}{ Drinker } \\
\hline Yes & 49 & 23 & 26 & 0.18 \\
\hline No & 41 & 22 & 19 & \\
\hline \multicolumn{5}{|l|}{ Metastasis } \\
\hline Yes & 51 & 43 & 8 & 0.10 \\
\hline No & 39 & 2 & 37 & \\
\hline
\end{tabular}

UCA1, urothelial cancer associated 1 .

healthy tissues in the majority of patients with LSCC. In addition, serum levels of lncRNA UCA1 were significantly higher in patients with LSCC compared with healthy controls. These data indicated that the upregulation of IncRNA UCA1 may be involved in LSCC.

The development of human diseases is usually accompanied with the changes in the levels of certain substances in the blood, and detecting the changes in blood levels of those substances may provide valuable information for the diagnosis and prognosis of certain diseases (17). Increased blood levels of UCA1 have indicated promising diagnostic and prognostic values for several types of cancer, including carcinoma of the urinary bladder (18) and gastric cancer in the early stages (19). In the present study, receiver operating characteristic curve analysis revealed that serum levels of UCA1 could be used to effectively distinguish patients with LSCC from healthy controls. In addition, the overall survival rate of patients with high serum levels of UCA1 was significantly lower than that of patients with low serum levels of UCA1. These data suggested that serum UCA1 may serve as a potential diagnostic and prognostic marker for LSCC. Previous studies have demonstrated that the expression of specific lncRNAs is affected by the individual's lifestyle, including smoking (20) and alcohol consumption (21), which may potentially impact the diagnostic and/or prognostic values of those lncRNAs for certain diseases. In the present study, serum levels of UCA1 were not significantly associated with sex, age and patients' lifestyle, including smoking and drinking. These data suggested that serum UCA1 may serve as a reliable and effective diagnostic and prognostic marker for LSCC. Additionally, smoking and drinking have been indicated to be risk factors for LSCC (2). Therefore, participation of UCA1 in pathogenesis of LSCC may not be achieved through smoking and drinking-induced pathways. Notably, UCA1 is a well-characterized lncRNA and its

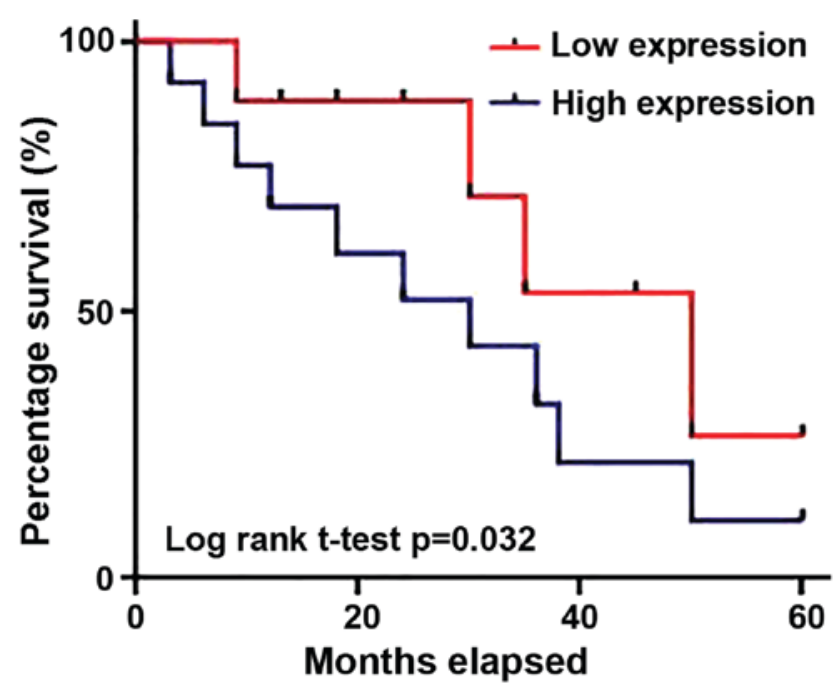

Figure 3. Survival curves of patients with laryngeal squamous cell carcinoma with high and low serum levels of urothelial cancer associated 1.

altered expression has been determined in a variety of human diseases, including lung cancer (16) and gastric cancer (19). These diseases should be excluded in the diagnosis and prognosis of LSCC or multiple markers should be used to improve the diagnosis and prognosis of LSCC using UCA1.

The Wnt/ $\beta$-catenin signal pathway is a key pathway involved in the pathogenesis of various human diseases (22), and the activation of $\mathrm{Wnt} / \beta$-catenin is common in different types of cancer (23). Activation of the Wnt/ $\beta$-catenin signaling pathway has been indicated to be responsible for the increased proliferation, migration and invasion abilities of LSCC cells, and inhibition of the $\mathrm{Wnt} / \beta$-catenin signaling pathway is considered to be a promising target to improve conditions of patients with LSCC (24). Recent studies have revealed that UCA1 can 

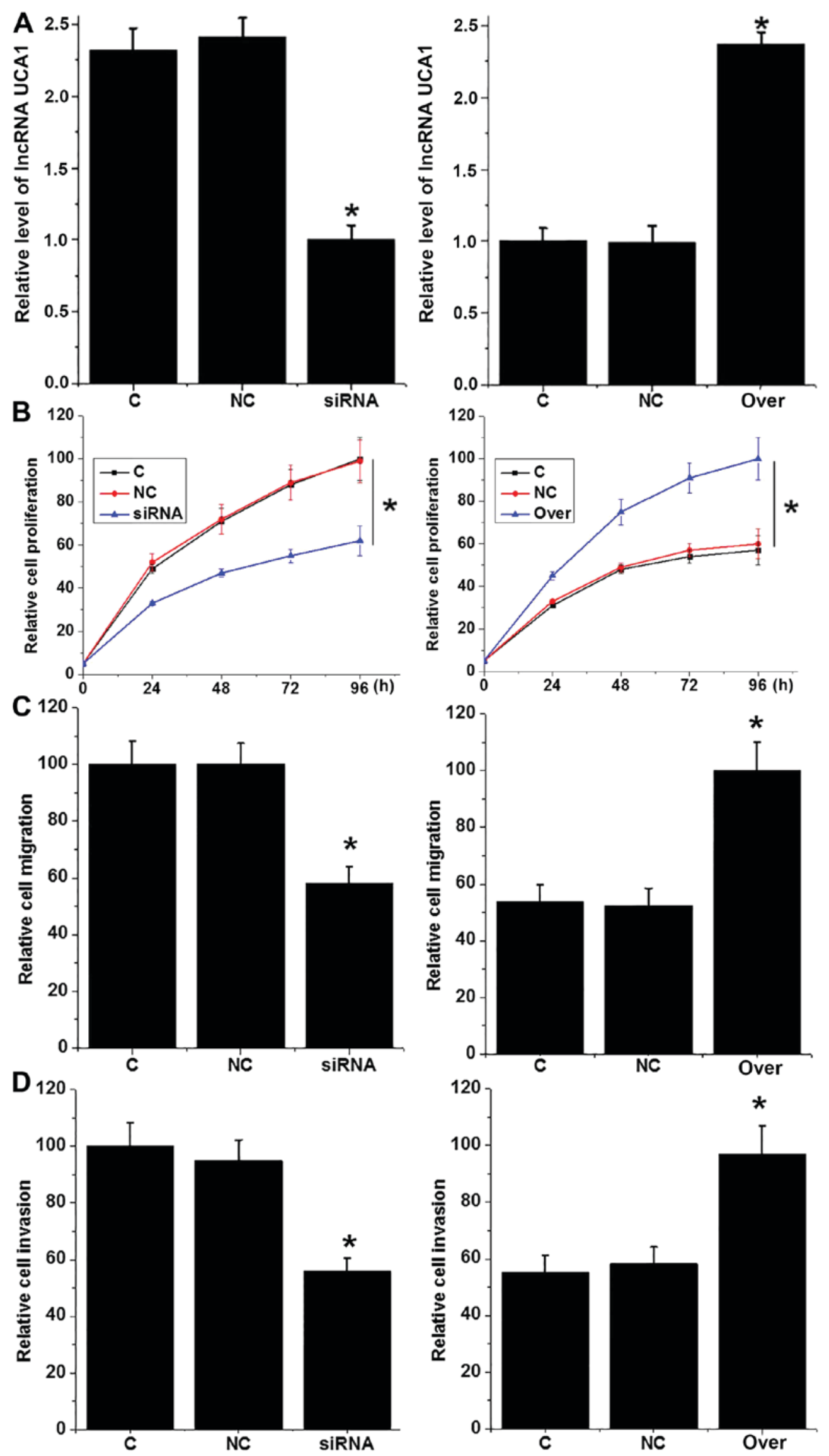

Figure 4. Effects of UCA1 knockdown and overexpression on proliferation, migration and invasion of LSCC cell line AMC-HN-8. (A) UCA1 knockdown and overexpression was confirmed by reverse transcription-quantitative polymerase chain reaction; Effects of UCA1 knockdown and overexpression on (B) proliferation, (C) migration and (D) invasion of LSCC cells. ${ }^{*} \mathrm{P}<0.05$, compared with control cells and negative control cells (C) and negative control (NC) cells. UCA1, urothelial cancer associated 1; LSCC, laryngeal squamous cell carcinoma; siRNA, small interfering RNA; Over, overexpression; NC, transfection with Silencer Select Negative Control No. 2 siRNA or empty pIRSE2-EGFP vector. 

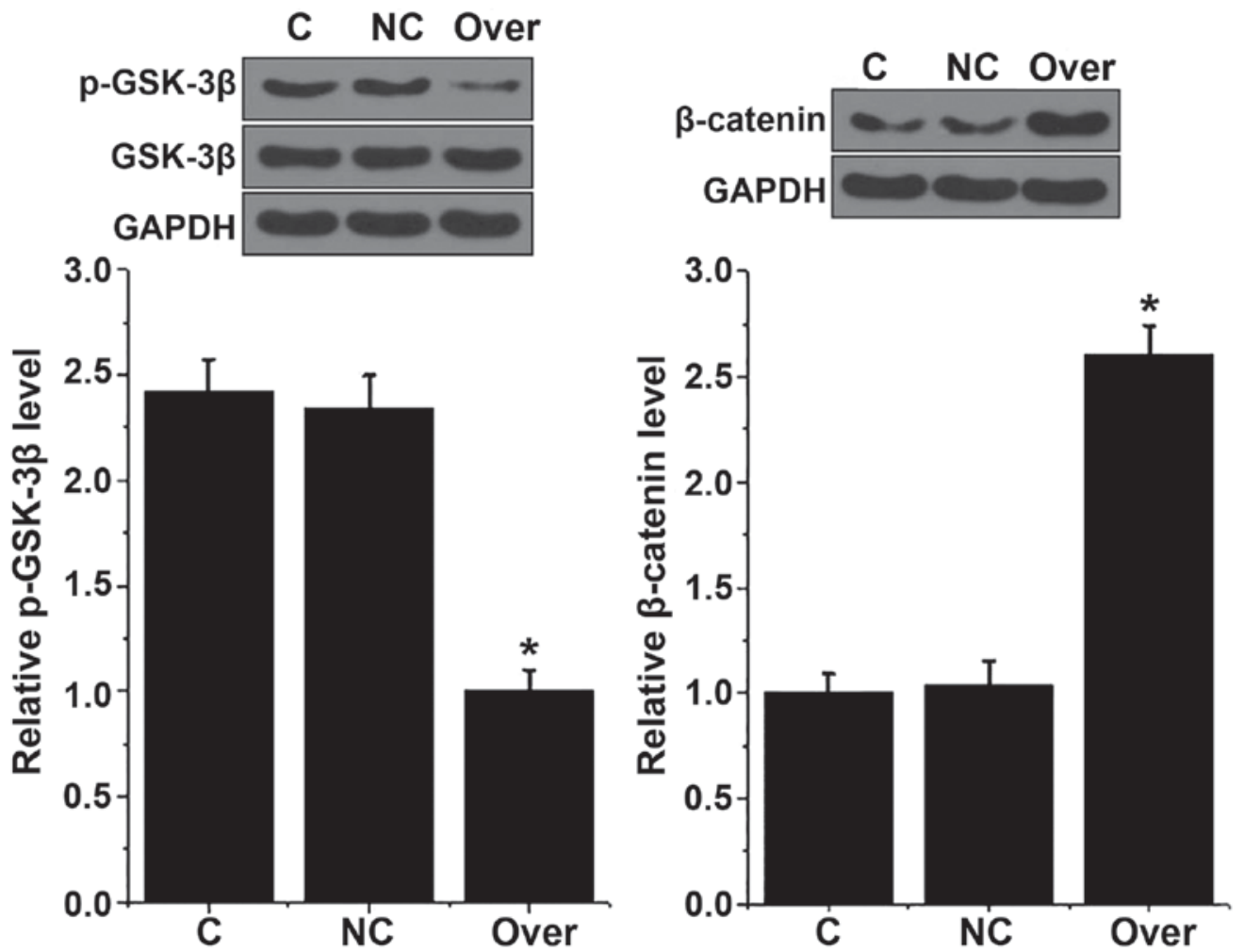

Figure 5. Effects of urothelial cancer associated 1 overexpression on Wnt/ $\beta$-catenin signaling pathway-associated proteins in AMC-HN-8 cells. ${ }^{*} \mathrm{P}<0.05$, compared with control cells and negative control cells. NC, transfection with empty pIRSE2-EGFP vector. GSK-3 $\beta$, glycogen synthase kinase-3 $\beta$; Over, overexpression; $\mathrm{C}$, control; $\mathrm{NC}$, negative control.
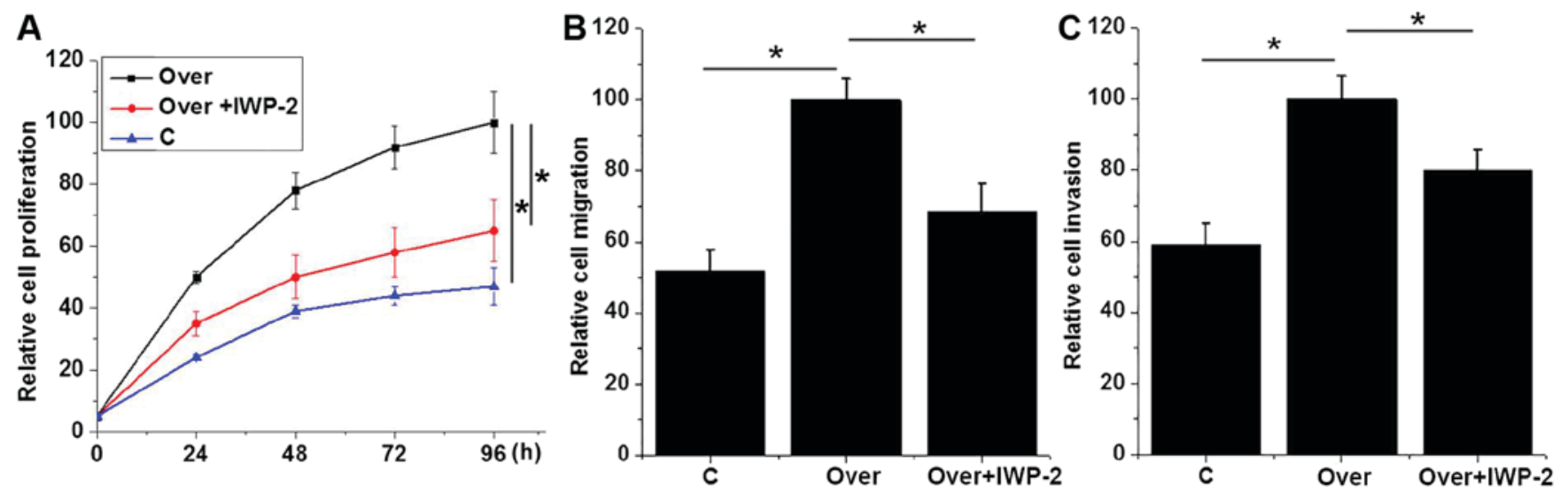

Figure 6. Effects of Wnt inhibitor IWP-2 on proliferation, migration and invasion of UCA1-overexpressed AMC-HN-8 cell line. Effects of Wnt inhibitor IWP-2 on (A) proliferation, (B) migration and (C) invasion of UCA1-overexpressed AMC-HN-8 cell line. " $\mathrm{P}<0.05$. UCA1, urothelial cancer associated 1; Over, overexpression; C, control; $\mathrm{NC}$, negative control.

interact with the Wnt/ $\beta$-catenin signaling pathway to achieve it pathological functions in different disease models $(9,25)$. In the present study, UCA1 overexpression inhibited the activation of a negative regulator of the $\mathrm{Wnt} / \beta$-catenin signaling pathway and also promoted the expression of $\beta$-catenin. Furthermore, treatment with Wnt inhibitor reduced the enhancing effects of UCA1 overexpression on proliferation, migration and invasion of LSCC cells. These data suggest that UCA1 can promote cell proliferation, invasion and migration of LSCC cells, at least partially by activating the $\mathrm{Wnt} / \beta$-catenin signaling pathway.

In conclusion, expression levels of UCA1 were significantly upregualted in tumor tissues compared with adjacent healthy tissues in the majority of patients with LSCC. Serum levels of UCA1 were also significantly increased in patients with LSCC compared with healthy controls. UCA1 overexpression and knockdown increased and reduced the proliferation, migration and invasion abilities of LSCC cells, respectively. Furthermore, UCA1 overexpression activated the Wnt/ $\beta$-catenin signaling pathway in LSCC cells. Notably, treatment with Wnt inhibitor reduced the enhancing effects of UCA1 overexpression on proliferation, migration and invasion of LSCC cells. Therefore, it was concluded that UCA1 can promote cell proliferation, invasion and migration of LSCC cells by activating the Wnt/ $\beta$-catenin signaling pathway. However, the present study 
is limited by the small sample size. For example, survival rate between high and low UCA1 expression groups became similar after the 5-year follow-up. Future studies with a larger sample size are required to further confirm the conclusions in the present study.

\section{Acknowledgements}

Not applicable.

\section{Funding}

No funding was received.

\section{Availability of data and materials}

The datasets used and/or analyzed during the current study are available from the corresponding author on reasonable request.

\section{Authors' contributions}

SS and KY designed the current study. SS and CG performed the experiments. SS and KY analyzed the data. KY interperted the data and drafted the manuscript. All authors approved the final version of the manuscript.

\section{Ethics approval and consent to participate}

The present study was approved by the Ethics Committee of the Central Hospital of Wuhan (Wuhan, China). All patients and healthy volunteers provided written informed consent prior to their inclusion within the study.

\section{Patient consent for publication}

Not applicable.

\section{Competing interests}

The authors declare that they have no competing interests.

\section{References}

1. Jemal A, Bray F, Center MM, Ferlay J, Ward E and Forman D: Global cancer statistics. CA Cancer J Clin 61: 69-90, 2011.

2. Wei Q, Yu D, Liu M, Wang M, Zhao M, Liu M, Jia W, Ma H, Fang J, Xu W, et al: Genome-wide association study identifies three susceptibility loci for laryngeal squamous cell carcinoma in the Chinese population. Nat Genet 46: 1110-1114, 2014.

3. Varalakshmi KP, Naik VS, Swapna RS, Sravani P and Neeraja M: Laryngeal biopsies with special reference to malignant tumors: A histopathological study. Int J Sci Study 4: 197-202, 2016.

4. Perkel JM: Visiting 'noncodarnia'. Biotechniques 54: 301, 303-304, 2013

5. Esteller M: Non-coding RNAs in human disease. Nat Rev Genet 12: 861-874, 2011.

6. Huarte M: The emerging role of lncRNAs in cancer. Nat Med 21: 1253-1261, 2015.

7. Han Y, Yang Y, Yuan H, Zhang TT, Sui H, Wei XL, Liu L, Huang P, Zhang WJ and Bai YX: UCA1, a long non-coding RNA up-regulated in colorectal cancer influences cell proliferation, apoptosis and cell cycle distribution. Pathology 46: 396-401, 2014
8. Yang N, Hui L, Wang Y, Yang H and Jiang X: Overexpression of SOX2 promotes migration, invasion, and epithelial-mesenchymal transition through the $\mathrm{Wnt} / \beta$-catenin pathway in laryngeal cancer AMC-HN-8 cells. Tumor Biol 35: 7965-7973, 2014.

9. Yang YT, Wang YF, Lai JY, Shen SY, Wang F, Kong J, Zhang W and Yang HY: Long non-coding RNA UCA1 contributes to the progression of oral squamous cell carcinoma by regulating the WNT/ $\beta$-catenin signaling pathway. Cancer Sci 107: 1581-1589, 2016.

10. Livak KJ and Schmittgen TD: Analysis of relative gene expression data using real-time quantitative PCR and the 2(-Delta Delta C(T)) method. Methods 25: 402-408, 2001.

11. Hanley JA and McNeil BJ: The meaning and use of the area under a receiver operating characteristic (ROC) curve. Radiology 143: 29-36, 1982.

12. Huang J, Zhou N, Watabe K, Lu Z, Wu F, Xu M and Mo YY: Long non-coding RNA UCA1 promotes breast tumor growth by suppression of p27 (Kip1). Cell Death Dis 5: e1008, 2015.

13. Tian Y, Zhang X, Hao Y, Fang Z and He Y: Potential roles of abnormally expressed long noncoding RNA UCA1 and Malat-1 in metastasis of melanoma. Melanoma Res 24: 335-341, 2014.

14. Hardisson D: Molecular pathogenesis of head and neck squamous cell carcinoma. Eur Arch Otorhinolaryngol 260: 502-508, 2003.

15. Shen Z, Li Q, Deng H, Lu D, Song H and Guo J: Long non-coding RNA profiling in laryngeal squamous cell carcinoma and its clinical significance: Potential biomarkers for LSCC. PLoS One 9: e108237, 2014.

16. Nie W, Ge HJ, Yang XQ, Sun X, Huang H, Tao X, Chen WS and $\mathrm{Li}$ B: LncRNA-UCA1 exerts oncogenic functions in non-small cell lung cancer by targeting miR-193a-3p. Cancer Lett 371: 99-106, 2016.

17. Burgdorf SK, Claesson MH, Nielsen HJ and Rosenberg J: Changes in cytokine and biomarker blood levels in patients with colorectal cancer during dendritic cell-based vaccination. Acta Oncol 48: 1157-1164, 2009.

18. Srivastava AK, Singh PK, Rath SK, Dalela D, Goel MM and Bhatt ML: Appraisal of diagnostic ability of UCA1 as a biomarker of carcinoma of the urinary bladder. Tumor Biol 35: 11435-11442, 2014.

19. Gao J, Cao R and Mu H: Long non-coding RNA UCA1 may be a novel diagnostic and predictive biomarker in plasma for early gastric cancer. Int J Clin Exp Pathol 8: 12936-12942, 2015.

20. Wang J, Qiu M, Xu Y, Li M, Dong G, Mao Q, Yin R and $\mathrm{Xu}$ L: Long noncoding RNA CCAT2 correlates with smoking in esophageal squamous cell carcinoma. Tumor Biol 36: 5523-5528, 2015.

21. Yu V, Singh P, Rahimy E, Zheng H, Kuo SZ, Kim E, Wang-Rodriguez J and Ongkeko WM: RNA-seq analysis identifies key long non-coding RNAs connected to the pathogenesis of alcohol-associated head and neck squamous cell carcinoma. Oncol Lett 12: 2846-2853, 2016.

22. Clevers $\mathrm{H}$ and Nusse $\mathrm{R}$ : Wnt/ $\beta$-catenin signaling and disease. Cell 149: 1192-1205, 2012.

23. Nagaraj AB, Joseph P, Kovalenko O, Singh S, Armstrong A, Redline R, Resnick K, Zanotti K, Waggoner S and DiFeo A: Critical role of $\mathrm{Wnt} / \beta$-catenin signaling in driving epithelial ovarian cancer platinum resistance. Oncotarget 6: 23720-23734, 2015.

24. Yang N, Wang Y, Hui L, Li X and Jiang X: SOX 1, contrary to SOX 2, suppresses proliferation, migration, and invasion in human laryngeal squamous cell carcinoma by inhibiting the Wnt/ß-catenin pathway. Tumor Biol 36: 8625-8635, 2015.

25. Liu H, Wang G, Yang L, Qu J, Yang Z and Zhou X: Knockdown of long non-coding RNA UCA1 increases the tamoxifen sensitivity of breast cancer cells through inhibition of Wnt/ $\beta$-catenin pathway. PLoS One 11: e0168406, 2016.

(i) $($ This work is licensed under a Creative Commons Attribution-NonCommercial-NoDerivatives 4.0 International (CC BY-NC-ND 4.0) License. 